Research Article

\title{
New Reproducing Kernel Functions
}

\begin{abstract}
Ali Akgül
Department of Mathematics, Faculty of Education, Dicle University, 21280 Diyarbakır, Turkey

Correspondence should be addressed to Ali Akgül; aliakgul00727@gmail.com

Received 18 November 2014; Revised 10 January 2015; Accepted 21 January 2015

Academic Editor: Masoud Hajarian

Copyright (C) 2015 Ali Akgül. This is an open access article distributed under the Creative Commons Attribution License, which permits unrestricted use, distribution, and reproduction in any medium, provided the original work is properly cited.

Some new reproducing kernel functions on time scales are presented. Reproducing kernel functions have not been found on time scales till now. These functions are very important on time scales and they will be very useful for researchers. We need these functions to solve dynamic equations on time scales with the reproducing kernel method.
\end{abstract}

\section{Introduction}

A time scale (which is a special case of a measure chain) is an arbitrary nonempty closed subset of the real numbers. Thus,

$$
\mathbb{R}, \mathbb{Z}, \mathbb{N}, \mathbb{N}_{0}
$$

that is, the real numbers, the integers, the natural numbers, and the nonnegative integers are examples of time scales, as are

$$
[0,1] \cup[2,3], \quad[0,1] \cup \mathbb{N} \text {, and the Cantor set, }
$$

while

$$
\mathbb{Q}, \mathbb{R} \backslash \mathbb{C}, \mathbb{C},(0,1)
$$

that is, the rational numbers, the irrational numbers, the complex numbers, and the open interval between 0 and 1 , are not time scales. The theory of time scales was presented by Stefan Hilger in order to unify continuous and discrete analysis [1]. For more details of time scales see [2].

A time scale is denoted by $\mathbb{T}$ in this work. The delta derivative $f^{\Delta}$ for a function $f$ defined on $\mathbb{T}$ is given as [1] follows:

(i) $f^{\Delta}=f^{\prime}$ is the usual derivative if $\mathbb{T}=\mathbb{R}$;

(ii) $f^{\Delta}=\Delta f$ is the usual forward difference operator if $\mathbb{T}=\mathbb{Z}$

Reproducing kernel functions on time scale $\mathbb{T}$ have been found in this work. These new reproducing kernel functions correspond to old reproducing kernel functions when $\mathbb{T}=\mathbb{R}$.
The paper is organized as follows. The history of the reproducing kernel method (RKM) is given in Section 2. Section 3 introduces several reproducing kernel spaces for differential equations. Section 4 is devoted to the reproducing kernel functions on time scales. Derivation of the reproducing kernel Hilbert space on time scales is presented in Section 5. It has been proved that the new reproducing kernel functions on time scales coincide with the old reproducing kernel functions when $\mathbb{T}=\mathbb{R}$ in Section 6. Examples are illustrated in Section 7. There are some conclusions in Section 8.

\section{History of Reproducing Kernel Method}

In this section, the history of RKM will be investigated. Reproducing kernel space is a special Hilbert space. In recent years, there are many papers on the solution of the nonlinear problems with RKM [3]. The concept of the reproducing kernel can be traced back to the paper of Zaremba [4] in 1908. It was proposed for discussing the boundary value problems of the harmonic functions. In the early development stage of the reproducing kernel theory, most of the works were applied by Bergman [5]. Bergman asserted the corresponding kernels of the harmonic functions with one or several variables and the corresponding kernel of the analytic function in squared metric and applied them in the research of the boundary value problem of the elliptic partial differential equation. This is the first stage in the development history of the reproducing kernel [3]. 
The second development stage of the reproducing kernel theory was started by Mercer [6]. Mercer discovered that the continuous kernel of the positive definite integral equation has the positive definite property [3]

$$
\sum_{i, j=1}^{n} k\left(x_{i}, y_{j}\right) \xi_{i} \xi_{j} \geq 0
$$

He named the kernel with this property as positive definite Hermite matrix. He also found out that the positive defined Hermite matrix corresponded to a function family, proposed a Hilbert space with inner product $\langle f, g\rangle$, and proved the reproducibility of the kernel in this space:

$$
u(y)=\langle u(x), k(x, y)\rangle .
$$

The third development stage of the reproducing kernel theory is related to Aronszajn [7]. In 1950, he reduced the works of the formers and studied a systematic reproducing kernel theory including the Bergman kernel function.

RKM, which accurately computes the series solution, is of great interest to applied sciences. Recently, a lot of research work has been devoted to the application of RKM to a wide class of stochastic and deterministic problems involving fractional differential equation, nonlinear oscillator with discontinuity, singular nonlinear two-point periodic boundary value problems, integral equations, and nonlinear partial differential equations [3]. The efficiency of RKM has been used by many authors to investigate several scientific applications. Geng and Cui [8] and Zhou et al. [9] applied RKM to handle second-order boundary value problems. Yao and Lin [10] and Wang et al. [11] investigated a class of singular boundary value problems by RKM. In [12], RKM was used to solve nonlinear infinite-delay-differential equations. Wang and Chao [13] and Zhou et al. [9] independently employed RKM to variable-coefficient partial differential equations. Geng and Cui [14] and Du and Cui [15] investigated the approximate solution of the forced Duffing equation with integral boundary conditions by combining the homotopy perturbation method and RKM. Lv and Cui [16] presented a new algorithm to solve linear fifth-order boundary value problems. In [17], the authors developed a new existence proof of solutions for nonlinear boundary value problems. Cui and $\mathrm{Du}$ [18] obtained the representation of the exact solution for nonlinear Volterra-Fredholm integral equations by using RKM. Wu and Li [19] applied an iterative reproducing kernel method to obtain the analytical approximate solution of a nonlinear oscillator with discontinuities. Recently, RKM was applied to fractional partial differential equations and multipoint boundary value problems [17]. For more details about RKM and the modified forms and its effectiveness, see $[20]$ and the references therein.

\section{Reproducing Kernel Functions for Differential Equations}

In this section, we define some useful reproducing kernel functions for differential equations.
Definition 1 (reproducing kernel function). Let $E \neq \emptyset$. A function $K: E \times E \rightarrow \mathbb{C}$ is called a reproducing kernel function of the Hilbert space $H$ if and only if

(a) $K(\cdot, t) \in H$ for all $t \in E$,

(b) $\langle\varphi, K(\cdot, t)\rangle=\varphi(t)$ for all $t \in E$ and all $\varphi \in H$.

The last condition is called "the reproducing property" as the value of the function $\varphi$ at the point $t$ is reproduced by the inner product of $\varphi$ with $K(\cdot, t)$.

Definition 2 (reproducing kernel Hilbert space). A Hilbert space $H$ which is defined on a nonempty set $E$ is called a reproducing kernel Hilbert space if there exists a reproducing kernel function $K: E \times E \rightarrow \mathbb{C}$.

Definition 3. We define the space $G_{2}^{1}[a, b]$ by

$$
G_{2}^{1}[a, b]=\left\{u \in A C[a, b]: u^{\prime} \in L^{2}[a, b]\right\},
$$

where $A C$ denotes the space of absolutely continuous functions. The inner product and the norm in $G_{2}^{1}[a, b]$ are defined by

$$
\begin{gathered}
\langle u, v\rangle_{G_{2}^{1}}=u(a) v(a)+\int_{a}^{b} u^{\prime}(x) v^{\prime}(x) \mathrm{d} x, \quad u, v \in G_{2}^{1}[a, b], \\
\|u\|_{G_{2}^{1}}=\sqrt{\langle u, u\rangle_{G_{2}^{1}}}, \quad u \in G_{2}^{1}[a, b] .
\end{gathered}
$$

Theorem 4. The space $G_{2}^{1}[a, b]$ is a reproducing kernel space, and its reproducing kernel function $\widetilde{Q}_{y}$ is given by

$$
\widetilde{Q}_{y}(x)= \begin{cases}\sum_{i=1}^{2} c_{i}(y) x^{i-1}, & a \leq x \leq y \leq b, \\ \sum_{i=1}^{2} d_{i}(y) x^{i-1}, & a \leq y<x \leq b .\end{cases}
$$

Proof. By Definition 3, we have

$$
\left\langle u, \widetilde{Q}_{y}\right\rangle_{G_{2}^{1}}=u(a) \widetilde{Q}_{y}(a)+\int_{a}^{b} u^{\prime}(x) \widetilde{Q}_{y}^{\prime}(x) \mathrm{d} x .
$$

Integrating this equation by parts one time, we get

$$
\begin{aligned}
\left\langle u, \widetilde{Q}_{y}\right\rangle_{G_{2}^{1}}= & u(a) \widetilde{Q}_{y}(a)+u(b) \widetilde{Q}_{y}^{\prime}(b) \\
& -u(a) \widetilde{Q}_{y}^{\prime}(a)-\int_{a}^{b} u(x) \widetilde{Q}_{y}^{\prime \prime}(x) \mathrm{d} x .
\end{aligned}
$$

Note that property of the reproducing kernel is

$$
\left\langle u(x), \widetilde{Q}_{y}^{\prime}(x)\right\rangle_{G_{2}^{1}}=u(y) .
$$

If

$$
\begin{gathered}
\widetilde{Q}_{y}(a)-\widetilde{Q}_{y}^{\prime}(a)=0, \\
\widetilde{Q}_{y}^{\prime}(b)=0,
\end{gathered}
$$


then (9) gives

$$
-\widetilde{Q}_{y}^{\prime \prime}(x)=\delta(x-y)
$$

When $x \neq y$, we have

$$
\widetilde{Q}_{y}^{\prime \prime}(x)=0
$$

Therefore,

$$
\widetilde{Q}_{y}(x)= \begin{cases}c_{1}(y)+c_{2}(y) x, & a \leq x \leq y \leq b, \\ d_{1}(y)+d_{2}(y) x, & a \leq y<x \leq b .\end{cases}
$$

Since

$$
-\widetilde{Q}_{y}^{\prime \prime}(x)=\delta(x-y)
$$

we get

$$
\begin{gathered}
\widetilde{\mathrm{Q}}_{y^{+}}(y)=\widetilde{\mathrm{Q}}_{y^{-}}(y), \\
\widetilde{\mathrm{Q}}_{y^{+}}^{\prime}(y)-\widetilde{\mathrm{Q}}_{y^{-}}^{\prime}(y)=-1 .
\end{gathered}
$$

The unknown coefficients $c_{i}(y)$ and $d_{i}(y)(i=1,2)$ can be obtained by (12)-(18). Thus, $\widetilde{Q}_{y}$ is acquired as

$$
\widetilde{Q}_{y}(x)= \begin{cases}1+x-a, & a \leq x \leq y \leq b \\ 1+y-a, & a \leq y<x \leq b .\end{cases}
$$

Definition 5. We define the space $H_{2}^{2}[a, b]$ by

$$
H_{2}^{2}[a, b]=\left\{u \in A C[a, b]: u^{\prime} \in A C[a, b], u^{\prime \prime} \in L^{2}[a, b]\right\} .
$$

The inner product and the norm in $H_{2}^{2}[a, b]$ are defined by

$$
\begin{aligned}
&\langle u, v\rangle_{H_{2}^{2}}= u(a) v(a)+u^{\prime}(a) v^{\prime}(a) \\
&+\int_{a}^{b} u^{\prime \prime}(x) v^{\prime \prime}(x) \mathrm{d} x, \quad u, v \in H_{2}^{2}[a, b], \\
&\|u\|_{H_{2}^{2}}=\sqrt{\langle u, u\rangle_{H_{2}^{2}}}, \quad u \in H_{2}^{2}[a, b] .
\end{aligned}
$$

Theorem 6. The space $H_{2}^{2}[a, b]$ is a reproducing kernel space, and its reproducing kernel function $\widetilde{T}_{y}$ is given by

$$
\widetilde{T}_{y}(x)= \begin{cases}\sum_{i=1}^{4} c_{i}(y) x^{i-1}, & a \leq x \leq y \leq b, \\ \sum_{i=1}^{4} d_{i}(y) x^{i-1}, & a \leq y<x \leq b .\end{cases}
$$

Proof. By Definition 5, we have

$$
\begin{aligned}
\left\langle u, \widetilde{T}_{y}\right\rangle_{H_{2}^{2}}= & u(a) \widetilde{T}_{y}(a)+u^{\prime}(a) \widetilde{T}_{y}^{\prime}(a) \\
& +\int_{a}^{b} u^{\prime \prime}(x) \widetilde{T}_{y}^{\prime \prime}(x) \mathrm{d} x
\end{aligned}
$$

Integrating (23) by parts two times, we get

$$
\begin{aligned}
\left\langle u, \widetilde{T}_{y}\right\rangle_{H_{2}^{2}}= & u(a) \widetilde{T}_{y}(a)+u^{\prime}(a) \widetilde{T}_{y}^{\prime}(a) \\
& +u^{\prime}(b) \widetilde{T}_{y}^{\prime \prime}(b)-u^{\prime}(a) \widetilde{T}_{y}^{\prime \prime}(a) \\
& -u(b) \widetilde{T}_{y}^{\prime \prime \prime}(b)+u(a) \widetilde{T}_{y}^{\prime \prime \prime}(a) \\
& +\int_{a}^{b} u(x) \widetilde{T}_{y}^{(4)}(x) \mathrm{d} x .
\end{aligned}
$$

Note that property of the reproducing kernel is

$$
\left\langle u(x), \widetilde{T}_{y}(x)\right\rangle_{H_{2}^{2}}=u(y) \text {. }
$$

If

$$
\begin{gathered}
\widetilde{T}_{y}(a)+\widetilde{T}_{y}^{\prime \prime \prime}(a)=0, \\
\widetilde{T}_{y}^{\prime}(a)-\widetilde{T}_{y}^{\prime \prime}(a)=0, \\
\widetilde{T}_{y}^{\prime \prime}(b)=0, \\
\widetilde{T}_{y}^{\prime \prime \prime}(b)=0,
\end{gathered}
$$

then (23) gives

$$
\widetilde{T}_{y}^{(4)}(x)=\delta(x-y)
$$

When $x \neq y$, we get

$$
\widetilde{T}_{y}^{(4)}(x)=0
$$

Thus,

$$
\widetilde{Q}_{y}(x)=\left\{\begin{array}{r}
c_{1}(y)+c_{2}(y) x+c_{3}(y) x^{2}+c_{4}(y) x^{3}, \\
a \leq x \leq y \leq b, \\
d_{1}(y)+d_{2}(y) x+d_{3}(y) x^{2}+d_{4}(y) x^{3}, \\
a \leq y<x \leq b .
\end{array}\right.
$$

Since

$$
\widetilde{T}_{y}^{(4)}(x)=\delta(x-y)
$$

we have

$$
\begin{gathered}
\widetilde{T}_{y^{+}}^{(k)}(y)=\widetilde{T}_{y^{-}}^{(k)}(y), \quad k=0,1,2, \\
\widetilde{T}_{y^{+}}^{\prime \prime \prime}(y)-\widetilde{T}_{y^{-}}^{\prime \prime \prime}(y)=1 .
\end{gathered}
$$

The unknown coefficients $c_{i}(y)$ and $d_{i}(y)(i=1,2,3,4)$ can be obtained by (26)-(32). Thus, $\widetilde{T}_{y}$ is achieved as

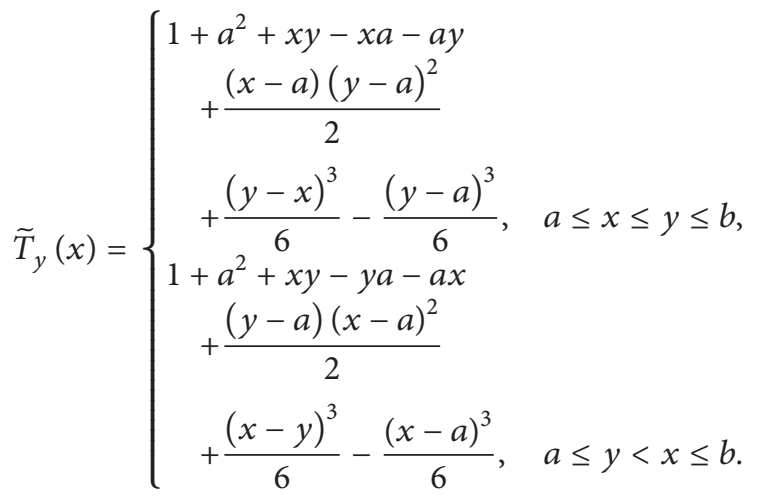


Definition 7. We define the space $W_{2}^{3}[a, b]$ by

$$
\begin{gathered}
W_{2}^{3}[a, b]=\left\{u \in A C[a, b]: u^{\prime}, u^{\prime \prime} \in A C[a, b],\right. \\
\left.u^{(3)} \in L^{2}[a, b]\right\} .
\end{gathered}
$$

The inner product and the norm in $W_{2}^{3}[a, b]$ are defined by

$$
\begin{gathered}
\langle u, v\rangle_{W_{2}^{3}}=\sum_{i=0}^{2} u^{(i)}(a) v^{(i)}(a)+\int_{a}^{b} u^{(3)}(x) v^{(3)}(x) \mathrm{d} x \\
u, v \in W_{2}^{3}[a, b] \\
\|u\|_{W_{2}^{3}}=\sqrt{\langle u, u\rangle_{W_{2}^{3}}}, \quad u \in W_{2}^{3}[a, b] .
\end{gathered}
$$

Theorem 8. The space $W_{2}^{3}[a, b]$ is a reproducing kernel space, and its reproducing kernel function $\widetilde{R}_{y}$ is given by

$$
\widetilde{R}_{y}(x)= \begin{cases}\sum_{i=1}^{6} c_{i}(y) x^{i-1}, & a \leq x \leq y \leq b, \\ \sum_{i=1}^{6} d_{i}(y) x^{i-1}, & a \leq y<x \leq b .\end{cases}
$$

Proof. By Definition 7, we get

$$
\left\langle u, \widetilde{R}_{y}\right\rangle_{W_{2}^{3}}=\sum_{i=0}^{2} u^{(i)}(a) \widetilde{R}_{y}^{(i)}(a)+\int_{a}^{b} u^{(3)}(x) \widetilde{R}_{y}^{(3)}(x) \mathrm{d} x .
$$

Integrating (37) by parts three times, we obtain

$$
\begin{aligned}
\left\langle u, \widetilde{R}_{y}\right\rangle_{W_{2}^{3}} & \\
= & u(a) \widetilde{R}_{y}(a)+u^{\prime}(a) \widetilde{R}_{y}^{\prime}(a)+u^{\prime \prime}(a) \widetilde{R}_{y}^{\prime \prime}(a) \\
& +u^{\prime \prime}(b) \widetilde{R}_{y}^{(3)}(b)-u^{\prime \prime}(a) \widetilde{R}_{y}^{(3)}(a) \\
& -u^{\prime}(b) \widetilde{R}_{y}^{(4)}(b)+u^{\prime}(a) \widetilde{R}_{y}^{(4)}(a)+u(b) \widetilde{R}_{y}^{(5)}(b) \\
& -u(a) \widetilde{R}_{y}^{(5)}(a) \\
& -\int_{a}^{b} u(x) \widetilde{R}_{y}^{(6)}(x) \mathrm{d} x .
\end{aligned}
$$

Note that property of the reproducing kernel is

$$
\left\langle u(x), \widetilde{R}_{y}(x)\right\rangle_{W_{2}^{3}}=u(y) .
$$

If

$$
\begin{gathered}
\widetilde{R}_{y}(a)-\widetilde{R}_{y}^{(5)}(a)=0, \\
\widetilde{R}_{y}^{\prime}(a)+\widetilde{R}_{y}^{(4)}(a)=0, \\
\widetilde{R}_{y}^{\prime \prime}(a)-\widetilde{R}_{y}^{(3)}(a)=0, \\
\widetilde{R}_{y}^{(3)}(b)=0, \\
\widetilde{R}_{y}^{(4)}(b)=0, \\
\widetilde{R}_{y}^{(5)}(b)=0 ;
\end{gathered}
$$

then, (37) gives

$$
-\widetilde{R}_{y}^{(6)}(x)=\delta(x-y) .
$$

When $x \neq y$, we know

$$
\widetilde{R}_{y}^{(6)}(x)=0 .
$$

Consequently, we attain

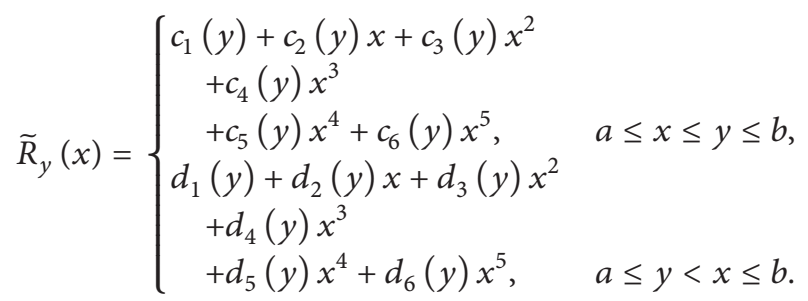

Since

$$
\widetilde{R}_{y}^{(6)}(x)=\delta(x-y),
$$

we have

$$
\begin{gathered}
\widetilde{R}_{y^{+}}^{(k)}(y)=\widetilde{R}_{y^{-}}^{(k)}(y), \quad k=0,1,2,3,4, \\
\widetilde{R}_{y^{+}}^{(5)}(y)-\widetilde{R}_{y^{-}}^{(5)}(y)=-1 .
\end{gathered}
$$

The unknown coefficients $c_{i}(y)$ and $d_{i}(y)(i=1,2,3,4,5,6)$ can be obtained by (40)-(46). Thus, $\widetilde{R}_{y}$ is gained as

$$
\widetilde{R}_{y}(x)=\left\{\begin{array}{cc}
1+x y+-a x-a y+a^{2} \\
+\frac{(x-y)^{5}}{120}-\frac{(a-y)^{5}}{120} \\
\quad-\frac{x(a-y)^{4}}{24}+\frac{a(a-y)}{24} \\
+\frac{(x-a)^{2}(y-a)^{2}}{4} \\
-\frac{(x-a)^{2}(a-y)^{3}}{12}, \\
+\frac{\left(y y+-a y-a x+a^{2}\right.}{120}-\frac{(a-x)^{5}}{120} \\
-\frac{y(a-x)^{4}}{24}+\frac{a(a-x)^{4}}{24} \\
+\frac{(y-a)^{2}(x-a)^{2}}{4}
\end{array}\right.
$$

\section{Reproducing Kernel Functions on Time Scales}

In this section, we define some useful reproducing kernel functions on time scale $\mathbb{T}$. All functions are new in the literature. 
Definition 9 (see $[1$, page 22]). A function $f: \mathbb{T} \rightarrow \mathbb{R}$ is called regulated provided its right-sided limits exist (finite) at all right-dense points in $\mathbb{T}$ and its left-sided limits exist (finite) at all left-dense points in $\mathbb{T}$.

Definition 10 (see [1, page 22]). A function $f: \mathbb{T} \rightarrow \mathbb{R}$ is called rd-continuous provided it is continuous at right-dense points in $\mathbb{T}$ and it is left-sided limits exist (finite) at left-dense points in $\mathbb{T}$. The set of rd-continuous functions $f: \mathbb{T} \rightarrow \mathbb{R}$ will be denoted by $C_{\mathrm{rd}}$.

Lemma 11 (see [1, page 28]). If $a, b, c \in \mathbb{T}, \alpha \in \mathbb{R}$, and $f, g \in$ $C_{r d}$, then

(i) $\int_{a}^{b}[f(t)+g(t)] \Delta t=\int_{a}^{b} f(t) \Delta t+\int_{a}^{b} g(t) \Delta t$;

(ii) $\int_{a}^{b}(\alpha f)(t) \Delta t=\alpha \int_{a}^{b} f(t) \Delta t$;

(iii) $\int_{a}^{b} f(t) \Delta t=-\int_{b}^{a} f(t) \Delta t$;

(iv) $\int_{a}^{b} f(t) \Delta t=\int_{a}^{c} f(t) \Delta t+\int_{c}^{b} f(t) \Delta t$;

(v) $\int_{a}^{b} f(\sigma(t)) g^{\Delta}(t) \Delta t=(f g)(b)-(f g)(a)-\int_{a}^{b} f^{\Delta}(t) g(t) \Delta t$;

(vi) $\int_{a}^{b} f(t) g^{\Delta}(t) \Delta t=(f g)(b)-(f g)(a)-\int_{a}^{b} f^{\Delta}(t) g(\sigma(t)) \Delta t$;

(vii) $\int_{a}^{a} f(t) \Delta t=0$;

(viii) if $|f(t)| \leq g(t)$ on $[a, b)$, then

$$
\left|\int_{a}^{b} f(t) \Delta t\right| \leq \int_{a}^{b} g(t) \Delta t
$$

(ix) if $f(t) \geq 0$ for all $a \leq t<b$, then $\int_{a}^{b} f(t) \Delta t \geq 0$.

Definition 12. We define the space $M$ as the set of all functions defined on $\mathbb{T}$. The inner product $\langle\cdot, \cdot\rangle_{1}$ and the norm $\|\cdot\|_{1}$ in $M$ are defined by

$$
\begin{gathered}
\langle u, v\rangle_{1}=u(a) v(a)+\int_{x=a}^{b} u^{\Delta}(x) v^{\Delta}(x) \Delta x, \quad u, v \in M, \\
\|u\|_{1}=\sqrt{\langle u, u\rangle_{1}}, \quad u \in M .
\end{gathered}
$$

Theorem 13. The space $\left(E,\langle\cdot, \cdot\rangle_{1}\right)$ is a reproducing kernel space, and its reproducing kernel function $Q_{y}$ is given by

$$
Q_{y}(x)= \begin{cases}1+y-a, & a \leq y<x \leq b \\ 1+x-a, & a \leq x \leq y \leq b\end{cases}
$$

Proof. Define $Q_{y}$ by (50) and note that

$$
Q_{y}^{\Delta}(x)= \begin{cases}0, & a \leq y<x \leq b \\ 1, & a \leq x \leq y \leq b .\end{cases}
$$

Let $u \in M$ and let $y \in[a, b]$. Then, by Definition 12 , we have

$$
\begin{aligned}
\left\langle u, Q_{y}\right\rangle_{1} & =u(a) Q_{y}(a)+\int_{x=a}^{b} u^{\Delta}(x) Q_{y}^{\Delta}(x) \Delta x \\
& =u(a)+\int_{x=a}^{y} u^{\Delta}(x) \Delta x \\
& =u(a)+u(y)-u(a) \\
& =u(y)
\end{aligned}
$$

This completes the proof.

Definition 14. The inner product $\langle\cdot, \cdot\rangle_{2}$ and the norm $\|\cdot\|_{2}$ in $M$ are defined by

$$
\begin{aligned}
\langle u, v\rangle_{2}= & u(a) v(a)+u^{\Delta}(a) v^{\Delta}(a) \\
& +\int_{x=a}^{b} u^{\Delta \Delta}(x) v^{\Delta \Delta}(x) \Delta x, \quad u, v \in M, \\
& \|u\|_{2}=\sqrt{\langle u, u\rangle_{2}}, \quad u \in M .
\end{aligned}
$$

Theorem 15. The space $\left(M,\langle\cdot, \cdot\rangle_{2}\right)$ is a reproducing kernel space, and its reproducing kernel function $T_{y}$ is given by

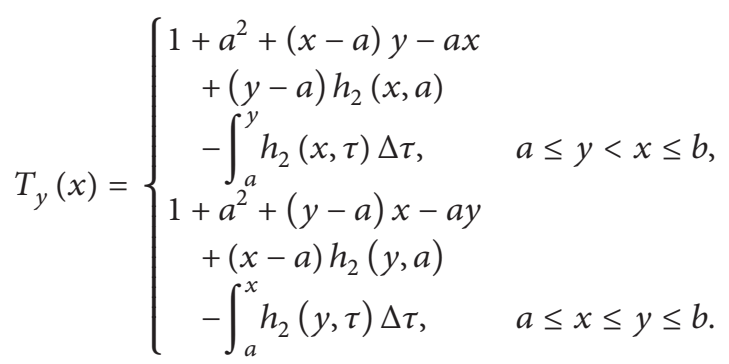

Proof. Define $T_{y}$ by (54) and note that

$$
\begin{aligned}
T_{y}^{\Delta}(x)= & \left\{\begin{array}{cc}
y-a+(y-a)(x-a) \\
-\int_{a}^{y} h_{1}(x, \tau) \Delta \tau, & a \leq y<x \leq b, \\
y-a-h_{2}(y, x)+h_{2}(y, a), & a \leq x \leq y \leq b .
\end{array}\right. \\
& T_{y}^{\Delta \Delta}(x)= \begin{cases}0, & a \leq y<x \leq b, \\
y-\sigma(x), & a \leq x \leq y \leq b .\end{cases}
\end{aligned}
$$

By Definition 14 and integration by parts, we have

$$
\begin{aligned}
\left\langle u, T_{y}\right\rangle_{2}= & u(a) T_{y}(a)+u^{\Delta}(a) T_{y}^{\Delta}(a) \\
& +\int_{x=a}^{b} u^{\Delta \Delta}(x) T_{y}^{\Delta \Delta}(x) \Delta x \\
= & u(a)\left(1+a^{2}+a(y-a)+a h_{2}(y, a)\right. \\
& \left.-a y-a h_{2}(y, a)\right)
\end{aligned}
$$




$$
\begin{aligned}
= & +u^{\Delta}(a)\left(y-a+h_{2}(y, a)-h_{2}(y, a)\right) \\
& -\int_{x=a}^{y}(y-\sigma(x)) u^{\Delta \Delta}(x) \Delta x \\
= & u(a)+(y-a) u^{\Delta}(a)+(y-y) u^{\Delta}(y) \\
& -(y-a) u^{\Delta}(a) \\
& -\int_{x=a}^{y}(-1) u^{\Delta}(x) \Delta x \\
= & u(a)+u(y)-u(a) \\
= & u(y) .
\end{aligned}
$$

This completes the proof.

Definition 16. The inner product $\langle\cdot, \cdot\rangle_{3}$ and the norm $\|\cdot\|_{3}$ in $M$ are defined by

$$
\begin{gathered}
\langle u, v\rangle_{3}=\sum_{i=0}^{2} u^{\Delta^{i}}(a) v^{\Delta^{i}}(a)+\int_{x=a}^{b} u^{\Delta \Delta \Delta}(x) v^{\Delta \Delta \Delta}(x) \Delta x, \\
u, v \in M, \\
\|u\|_{3}=\sqrt{\langle u, u\rangle_{3}}, \quad u \in M .
\end{gathered}
$$

Theorem 17. The space $\left(M,\langle\cdot, \cdot\rangle_{3}\right)$ is a reproducing kernel space, and its reproducing kernel function $R_{y}$ is given by

$$
\begin{aligned}
& R_{y}(x)
\end{aligned}
$$

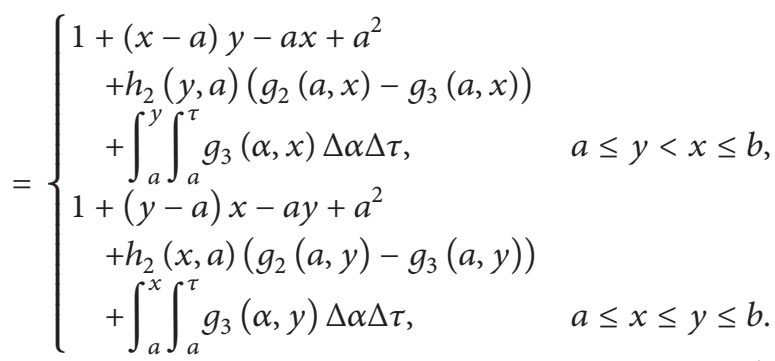

Proof. Define $R_{y}$ by (58) and note that

$$
\begin{aligned}
& R_{y}^{\Delta}(x)
\end{aligned}
$$

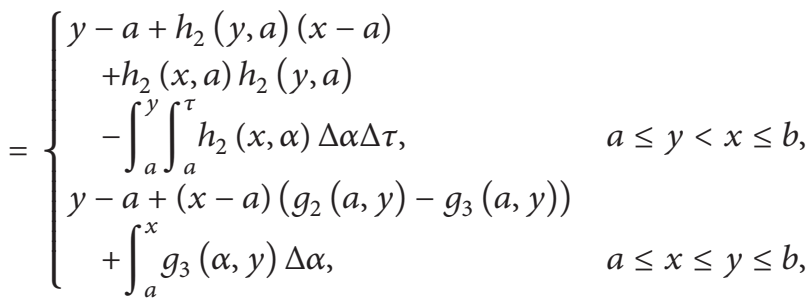

$$
\begin{gathered}
R_{y}^{\Delta \Delta}(x) \\
=\left\{\begin{array}{rr}
h_{2}(y, a)+(x-a) h_{2}(y, a) \\
-\int_{a}^{y} \int_{a}^{\tau}(x-\alpha) \Delta \alpha \Delta \tau, & a \leq y<x \leq b, \\
g_{2}(a, y)-g_{3}(a, y)+g_{3}(x, y), & a \leq x \leq y \leq b,
\end{array}\right. \\
R_{y}^{\Delta \Delta \Delta}(x)= \begin{cases}0, & a \leq y<x \leq b, \\
g_{2}(\sigma(x), y), & a \leq x \leq y \leq b .\end{cases}
\end{gathered}
$$

By Definition 16, we have

$$
\begin{aligned}
\left\langle u, R_{y}\right\rangle_{3} & \sum_{i=0}^{2} u^{\Delta^{i}}(a) R_{y}^{\Delta^{i}}(a)+\int_{x=a}^{b} u^{\Delta \Delta \Delta}(x) R_{y}^{\Delta \Delta \Delta}(x) \Delta x \\
= & u(a)\left(1+a y-a^{2}-a y+a^{2}\right)+u^{\Delta}(a)(y-a) \\
& +u^{\Delta \Delta}(a) g_{2}(a, y)+\int_{a}^{y} g_{2}(\sigma(x), y) u^{\Delta \Delta \Delta}(x) \Delta x \\
= & u(a)+(y-a) u^{\Delta}(a)+g_{2}(a, y) u^{\Delta \Delta}(a) \\
& +g_{2}(y, y) u^{\Delta \Delta}(y) \\
& -g_{2}(a, y) u^{\Delta \Delta}(a)-\int_{a}^{y} g_{1}(\sigma(x), y) u^{\Delta \Delta}(x) \Delta x \\
= & u(a)+(y-a) u^{\Delta}(a)-g_{1}(y, y) u^{\Delta}(y) \\
& +g_{1}(a, y) u^{\Delta}(a)+\int_{a}^{y} u^{\Delta}(x) \Delta x \\
= & u(a)+u(y)-u(a) \\
= & u(y) .
\end{aligned}
$$

This completes the proof.

\section{Derivation of Reproducing Kernel Hilbert Space on Time Scales}

Derivation of the new reproducing kernel functions are presented in this section. By Definition 9, we have

$$
\left\langle u, Q_{y}\right\rangle_{1}=u(a) Q_{y}(a)+\int_{x=a}^{b} u^{\Delta}(x) Q_{y}^{\Delta}(x) \Delta x
$$

We get

$$
\begin{aligned}
\left\langle u, Q_{y}\right\rangle_{1}= & u(a) Q_{y}(a)+u(b) Q_{y}(b)-u(a) Q_{y}(a) \\
& -\int_{x=a}^{y} u(\sigma(x)) Q_{y}^{\Delta \Delta}(x) \Delta x
\end{aligned}
$$


TABLE 1: Values of $Q_{y}$ by (63).

\begin{tabular}{llllll}
\hline$x / y$ & 0 & 1 & 2 & 3 & 4 \\
\hline 0 & 1 & 1 & 1 & 1 & 1 \\
1 & 1 & 2 & 2 & 2 & 2 \\
2 & 1 & 2 & 3 & 3 & 3 \\
3 & 1 & 2 & 3 & 4 & 4 \\
4 & 1 & 2 & 3 & 4 & 5 \\
\hline
\end{tabular}

by integration by parts. We have

$$
\begin{gathered}
Q_{y}(a)-Q_{y}^{\Delta}(a)= \begin{cases}1 & \text { if } y=a, \\
0 & \text { otherwise },\end{cases} \\
Q_{y}^{\Delta}(b)= \begin{cases}1 & \text { if } y=b, \\
0 & \text { otherwise, }\end{cases} \\
Q_{y}^{\Delta \Delta}(b)= \begin{cases}1 & \text { if } y=\sigma(x), \\
0 & \text { otherwise. }\end{cases}
\end{gathered}
$$

We acquire Table 1 by (63). We get

$$
Q_{y}(x)= \begin{cases}1+y-a, & a \leq y<x \leq b \\ 1+x-a, & a \leq x \leq y \leq b\end{cases}
$$

by Table $1, T_{y}$ and $R_{y}$ can be found in a similar way.

Theorem 18. The space $\left(M,\langle\cdot, \cdot\rangle_{3}\right)$ is a reproducing kernel Hilbert space.

Proof. Assume that $u_{n}$ is a Cauchy sequence in $\left(M,\langle\cdot, \cdot\rangle_{3}\right)$. Thus, we have

$$
\begin{aligned}
& \left\|u_{n+p}-u_{n}\right\|_{3}^{2} \\
& =\sum_{i=0}^{2}\left[u_{n+p}^{\Delta^{i}}(a)-u_{n}^{\Delta^{i}}(a)\right]^{2} \\
& \quad+\int_{x=a}^{b}\left[u_{n+p}^{\Delta \Delta \Delta}(x)-u_{n}^{\Delta \Delta \Delta}(x)\right]^{2} \Delta x \underset{n \rightarrow \infty}{\longrightarrow} 0 .
\end{aligned}
$$

Therefore, we acquire

$$
\begin{aligned}
& u_{n+p}^{\Delta^{i}}(a)-u_{n}^{\Delta^{i}}(a) \underset{n \rightarrow \infty}{\longrightarrow} 0, \quad i=0,1,2, \\
& \int_{x=a}^{b}\left[u_{n+p}^{\Delta \Delta \Delta}(x)-u_{n}^{\Delta \Delta \Delta}(x)\right]^{2} \Delta x \underset{n \rightarrow \infty}{\longrightarrow} 0
\end{aligned}
$$

which shows that for any $i(0 \leq i \leq 2)$, the sequence $u_{n}^{\left(\Delta^{i}\right)}(a)(n=1,2, \ldots)$ is a Cauchy sequence in $\mathbb{T}$ and $u_{n}^{\left(\Delta^{3}\right)}(x)(n=1,2, \ldots)$ is a Cauchy sequence in space $L^{2}[a, b]$. Consequently, there exist a unique number $\sigma_{i}(i=0,1,2)$ and a unique function $g(x) \in L^{2}[a, b]$, satisfying

$$
\begin{gathered}
\lim _{n \rightarrow \infty} u_{n}^{(i)}(a) \longrightarrow \sigma_{i} \quad(i=0,1,2), \\
\lim _{n \rightarrow \infty} \int_{x=a}^{b}\left[u_{n}^{\Delta \Delta \Delta}(x)-g(x)\right]^{2} \Delta x \longrightarrow 0 .
\end{gathered}
$$

Now, suppose that $x$ is right scattered. It can be seen that the sequence $u_{n}(x)$ is a Cauchy sequence and converges to $u(x) \epsilon$ $\left(M,\langle\cdot, \cdot\rangle_{3}\right)$. Hence,

$$
\left\|u-u_{n}\right\|_{3} \longrightarrow 0
$$

Therefore, $\left(M,\langle\cdot, \cdot\rangle_{3}\right)$ is a Hilbert space. This space is a reproducing kernel space by Definition 2. Consequently, $\left(M,\langle\cdot, \cdot\rangle_{3}\right)$ is a reproducing kernel Hilbert space.

Theorem 19. $\left(M,\langle\cdot, \cdot\rangle_{1}\right)$ and $\left(M,\langle\cdot, \cdot\rangle_{2}\right)$ are reproducing kernel Hilbert spaces.

Proof. The proof of this theorem is similar to the proof of the Theorem 18. Therefore, the proof is omitted.

Remark 20. Old reproducing kernel functions $\widetilde{Q}_{y}, \widetilde{T}_{y}, \widetilde{R}_{y}$ have been in the literature and many authors have used them to solve first-order and second-order differential equations. However, our new reproducing kernel functions $Q_{y}, T_{y}, R_{y}$ are new in the literature and no one has used them before. These new reproducing kernel functions will be used to solve dynamic equations on time scales by RKM in my forthcoming papers.

\section{Comparing Reproducing Kernel Functions When $\mathbb{T}=\mathbb{R}$}

(i) When $\mathbb{T}=\mathbb{R}$, it is obvious that

$$
\widetilde{Q}_{y}(x)=Q_{y}(x) .
$$

(ii) When $\mathbb{T}=\mathbb{R}$, we have

$$
g_{k}(t, s)=h_{k}(t, s)=\frac{(t-s)^{k}}{k !} \quad \forall s, t \in \mathbb{R} .
$$

Therefore,

$$
\begin{gathered}
h_{2}(x, a)=\frac{(x-a)^{2}}{2}, \\
\int_{a}^{y} h_{2}(y, \tau)=\frac{(x-y)^{3}}{6}-\frac{(x-a)^{3}}{6}, \\
h_{2}(y, a)=\frac{(y-a)^{2}}{2}, \\
\int_{a}^{x} h_{2}(y, \tau)=\frac{(y-x)^{3}}{6}-\frac{(y-a)^{3}}{6} .
\end{gathered}
$$

If we substitute (71) into the $T_{y}$, we get

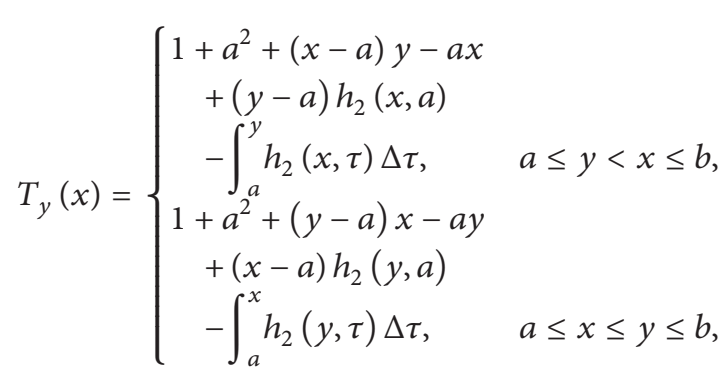




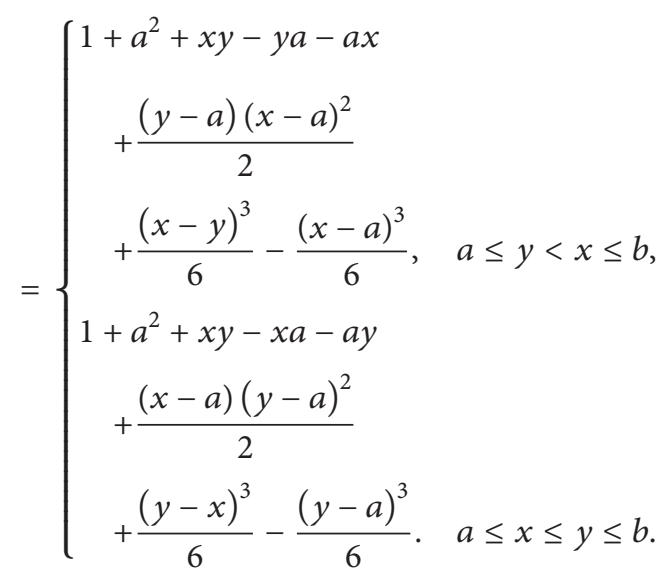

This shows that $T_{y}(x)=\widetilde{T}_{y}(x)$ when $\mathbb{T}=\mathbb{R}$.

(iii) When $\mathbb{T}=\mathbb{R}$, we know that

$$
\begin{gathered}
h_{2}(y, a)=\frac{(y-a)^{2}}{2}, \quad g_{2}(a, x)=\frac{(a-x)^{2}}{2}, \\
g_{3}(a, x)=\frac{(a-x)^{3}}{6}, \\
\int_{a}^{y} \int_{a}^{\tau} g_{3}(\alpha, x) \Delta \alpha \Delta \tau \\
=\frac{(x-y)^{5}}{120}-\frac{(a-y)^{5}}{120}-x \frac{(a-y)^{4}}{24}+a \frac{(a-y)^{4}}{24}, \\
h_{2}(x, a)=\frac{(x-a)^{2}}{2}, \quad g_{2}(a, y)=\frac{(a-y)^{2}}{2}, \\
\int_{a}^{x} \int_{a}^{\tau} g_{3}(\alpha, y) \Delta \alpha \Delta \tau \\
\left.=\frac{(y-x)^{5}}{120}-\frac{(a-x)^{5}}{120}-y \frac{(a-x)^{4}}{24}+a \frac{(a-x)^{4}}{24} . y\right)^{3} \\
6
\end{gathered}
$$

If we put (73) into the $R_{y}$, we acquire

$$
\begin{aligned}
& R_{y}(x)
\end{aligned}
$$

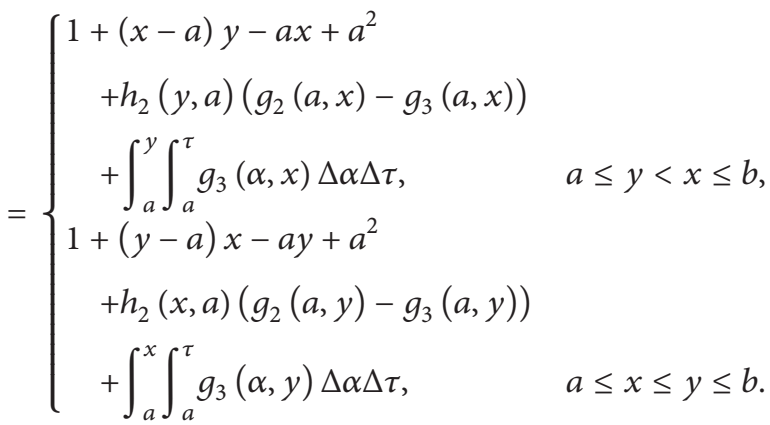

$$
\left\{\begin{array}{rl}
1+ & x y+-a y-a x+a^{2} \\
& +\frac{(y-x)^{5}}{120}-\frac{(a-x)^{5}}{120} \\
& -\frac{y(a-x)^{4}}{24}+\frac{a(a-x)^{4}}{24} \\
& +\frac{(y-a)^{2}(x-a)^{2}}{4} \\
& -\frac{(y-a)^{2}(a-x)^{3}}{12}, \\
1 & +x y+-a x-a y+a^{2} \\
& +\frac{(x-y)^{5}}{120}-\frac{(a-y)^{5}}{120} \\
& -\frac{x(a-y)^{4}}{24}+\frac{a(a-y)^{4}}{24} \\
& +\frac{(x-a)^{2}(y-a)^{2}}{4} \\
& -\frac{(x-a)^{2}(a-y)^{3}}{12},
\end{array} \quad a \leq x \leq b \leq b \leq b .\right.
$$

This proves that $R_{y}(x)=\widetilde{R}_{y}(x)$ when $\mathbb{T}=\mathbb{R}$.

\section{Examples}

In this section, common reproducing kernel functions will be obtained. These functions have been used to solve first and second order differential equations with RKM by many researchers. The function space $W_{2}^{m}[a, b]$ is defined as follows:

$$
\begin{aligned}
W_{2}^{m}[a, b]=\{ & f(x) \mid \text { is absolutely continuous, } \\
& \left.f^{(m)} \in L^{2}[a, b], x \in[a, b]\right\} .
\end{aligned}
$$

The inner product and the norm in the function space $W_{2}^{m}[a, b]$ are defined as follows:

$$
\begin{gathered}
\langle f, g\rangle=\sum_{i=0}^{m-1} f^{(i)}(a) g^{(i)}(a)+\int_{a}^{b} f^{(m)}(x) g^{(m)}(x) \mathrm{d} x, \\
\|f\|_{W_{2}^{m}}=\sqrt{\langle f, f\rangle_{W_{2}^{m}}}
\end{gathered}
$$

Function space $\mathrm{W}_{2}^{m}[a, b]$ is a reproducing kernel Hilbert space and its reproducing kernel function $V_{y}$ is given as [3]

$$
V_{y}(x)= \begin{cases}\sum_{i=1}^{2 m} c_{i}(y) x^{i-1}, & x<y, \\ \sum_{i=1}^{2 m} d_{i}(y) x^{i-1}, & x>y .\end{cases}
$$

Example 1 (see [3, page 17]). When we take $m=1, a=$ $0, b=1$, then we get

$$
V_{y}(x)= \begin{cases}1+x, & 0 \leq x \leq y \leq 1 \\ 1+y, & 0 \leq y<x \leq 1\end{cases}
$$

We acquire $V_{y}(x)=Q_{y}(x)$ at $\mathbb{T}=\mathbb{R}$. 
Example 2 (see [3, page 11]). When we choose $m=2, a=$ $0, b=1$, then we acquire

$$
\begin{aligned}
& V_{y}(x)= \begin{cases}1+x y+\frac{x^{2} y}{2}-\frac{x^{3}}{6}, & 0 \leq x \leq y \leq 1, \\
1+x y+\frac{y^{2} x}{2}-\frac{y^{3}}{6}, & 0 \leq y<x \leq 1,\end{cases} \\
& P_{y}(x)=\left\{\begin{array}{rr}
1+x y+y h_{2}(x, 0) \\
-\int_{0}^{y} h_{2}(x, \tau) \Delta \tau, & 0 \leq y<x \leq 1, \\
1+y x+x h_{2}(y, 0) & \\
-\int_{0}^{x} h_{2}(y, \tau) \Delta \tau, & 0 \leq x \leq y \leq 1 .
\end{array}\right.
\end{aligned}
$$

We obtain $V_{y}(x)=P_{y}(x)$ at $\mathbb{T}=\mathbb{R}$.

Example 3 (see [3, page 17]). If we take $m=3, a=0, b=1$, then we obtain

$$
V_{y}(x)=\left\{\begin{array}{cc}
1+x y+\frac{x^{2} y^{2}}{4}+\frac{x^{3} y^{2}}{12} & \\
-\frac{x^{4} y}{24}+\frac{x^{5}}{120}, & 0 \leq x \leq y \leq 1, \\
1+x y+\frac{x^{2} y^{2}}{4}+\frac{y^{3} x^{2}}{12} & \\
-\frac{y^{4} x}{24}+\frac{y^{5}}{120}, & 0 \leq y<x \leq 1,
\end{array}\right.
$$$$
F_{y}(x)
$$$$
=\left\{\begin{aligned}
1+ & x y+h_{2}(y, 0) \\
& \cdot\left(g_{2}(0, x)-g_{3}(0, x)\right) \\
& +\int_{0}^{y} \int_{0}^{\tau} g_{3}(\alpha, x) \Delta \alpha \Delta \tau, \quad 0 \leq y<x \leq 1, \\
1+ & y x+h_{2}(x, 0) \\
& \cdot\left(g_{2}(0, y)-g_{3}(0, y)\right) \\
& +\int_{0}^{x} \int_{0}^{\tau} g_{3}(\alpha, y) \Delta \alpha \Delta \tau, \quad 0 \leq x \leq y \leq 1 .
\end{aligned}\right.
$$

We get $V_{y}(x)=F_{y}(x)$ at $\mathbb{T}=\mathbb{R}$.

\section{Conclusion}

In this work, reproducing kernel functions on time scales were found. It was proved that reproducing kernel functions on time scale $\mathbb{T}$ coincide with the old reproducing kernel functions when $\mathbb{T}=\mathbb{R}$. These reproducing kernel functions are new on time scales. Therefore, they will be very useful to solve dynamic equations on time scales.

\section{Conflict of Interests}

The author declares that there is no conflict of interests regarding the publication of this paper.

\section{Acknowledgment}

The author would like to express his thanks to unknown referees for their careful reading and helpful comments. This paper is supported by Dicle University.

\section{References}

[1] M. Bohner and A. Peterson, Dynamic Equations on Time Scales: An Introduction with Applications, Birkhäuser, Boston, Mass, USA, 2001.

[2] R. P. Agarwal and D. O'Regan, "Triple solutions to boundary value problems on time scales," Applied Mathematics Letters, vol. 13, no. 4, pp. 7-11, 2000.

[3] M. Cui and Y. Lin, Nonlinear Numerical Analysis in the Reproducing Kernel Space, Nova Science, New York, NY, USA, 2009.

[4] S. Zaremba, "Sur le calcul numérique des fonctions demandées dans le problème de dirichlet et le problème hydrodynamique," Bulletin International de l'Académie des Sciences de Cracovie, vol. 68, pp. 125-195, 1908.

[5] S. Bergman, The Kernel Function and Conformal Mapping, American Mathematical Society, New York, NY, USA, 1950.

[6] J. Mercer, "Function of positive and negative type and their connection with the theory of integral equation," Philosophical Transactions of the Royal Society of London. Series B, vol. 209, pp. 415-446, 1909.

[7] N. Aronszajn, "Theory of reproducing kernels," Transactions of the American Mathematical Society, vol. 68, pp. 337-404, 1950.

[8] F. Geng and M. Cui, "Solving a nonlinear system of second order boundary value problems," Journal of Mathematical Analysis and Applications, vol. 327, no. 2, pp. 1167-1181, 2007.

[9] Y. Zhou, Y. Lin, and M. Cui, "An efficient computational method for second order boundary value problems of nonlinear differential equations," Applied Mathematics and Computation, vol. 194, no. 2, pp. 354-365, 2007.

[10] H. Yao and Y. Lin, "Solving singular boundary-value problems of higher even-order," Journal of Computational and Applied Mathematics, vol. 223, no. 2, pp. 703-713, 2009.

[11] Y. Wang, L. Su, X. Cao, and X. Li, "Using reproducing kernel for solving a class of singularly perturbed problems," Computers \& Mathematics with Applications, vol. 61, no. 2, pp. 421-430, 2011.

[12] X. Lü and M. Cui, "Analytic solutions to a class of nonlinear infinite-delay-differential equations," Journal of Mathematical Analysis and Applications, vol. 343, no. 2, pp. 724-732, 2008.

[13] Y.-L. Wang and L. Chao, "Using reproducing kernel for solving a class of partial differential equation with variable-coefficients," Applied Mathematics and Mechanics. English Edition, vol. 29, no. 1, pp. 129-137, 2008.

[14] F. Geng and M. Cui, "New method based on the HPM and RKHSM for solving forced Duffing equations with integral boundary conditions," Journal of Computational and Applied Mathematics, vol. 233, no. 2, pp. 165-172, 2009.

[15] J. Du and M. Cui, "Solving the forced Duffing equation with integral boundary conditions in the reproducing kernel space," International Journal of Computer Mathematics, vol. 87, no. 9, pp. 2088-2100, 2010.

[16] X. Lv and M. Cui, "An efficient computational method for linear fifth-order two-point boundary value problems," Journal of Computational and Applied Mathematics, vol. 234, no. 5, pp. 1551-1558, 2010. 
[17] W. Jiang and Y. Lin, "Representation of exact solution for the time-fractional telegraph equation in the reproducing kernel space," Communications in Nonlinear Science and Numerical Simulation, vol. 16, no. 9, pp. 3639-3645, 2011.

[18] M. Cui and H. Du, "Representation of exact solution for the nonlinear Volterra-Fredholm integral equations," Applied Mathematics and Computation, vol. 182, no. 2, pp. 1795-1802, 2006.

[19] B. Wu and X. Li, "Iterative reproducing kernel method for nonlinear oscillator with discontinuity," Applied Mathematics Letters, vol. 23, no. 10, pp. 1301-1304, 2010.

[20] M. Inc, A. Akgül, and A. Kılıçman, "A new application of the reproducing kernel Hilbert space method to solve MHD Jeffery-HAMel flows problem in nonparallel walls," Abstract and Applied Analysis, vol. 2013, Article ID 239454, 12 pages, 2013. 


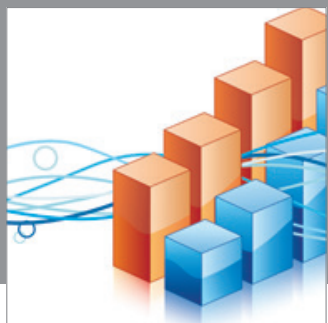

Advances in

Operations Research

mansans

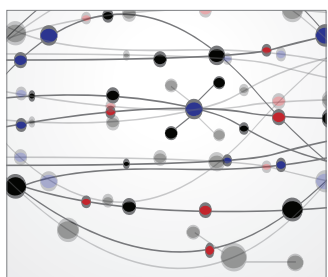

The Scientific World Journal
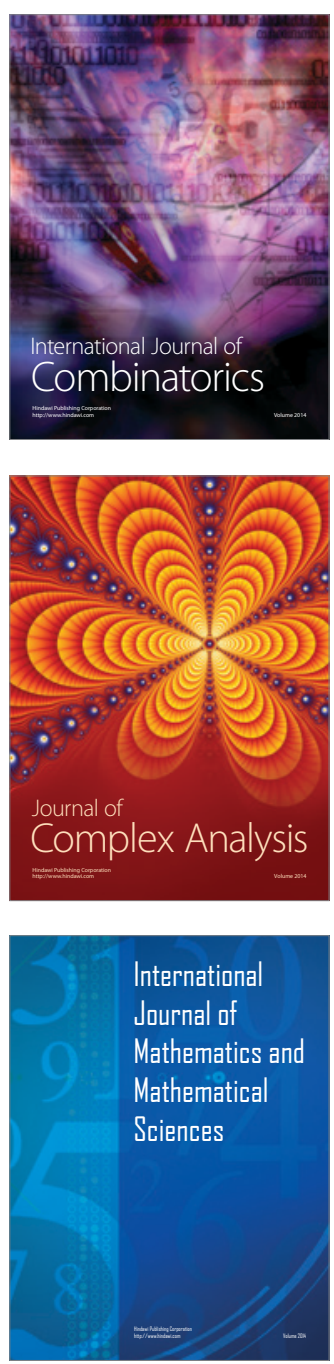
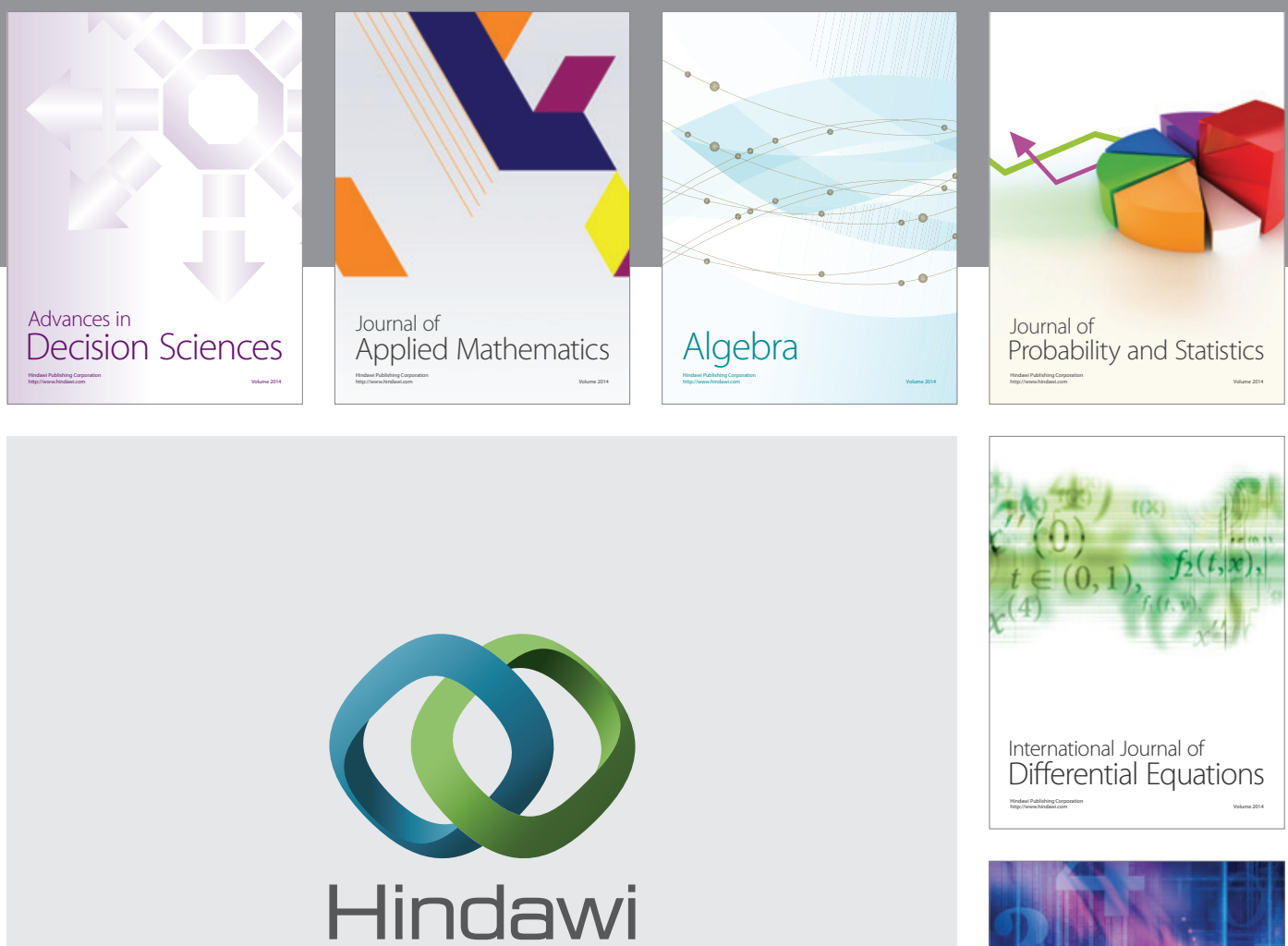

Submit your manuscripts at http://www.hindawi.com
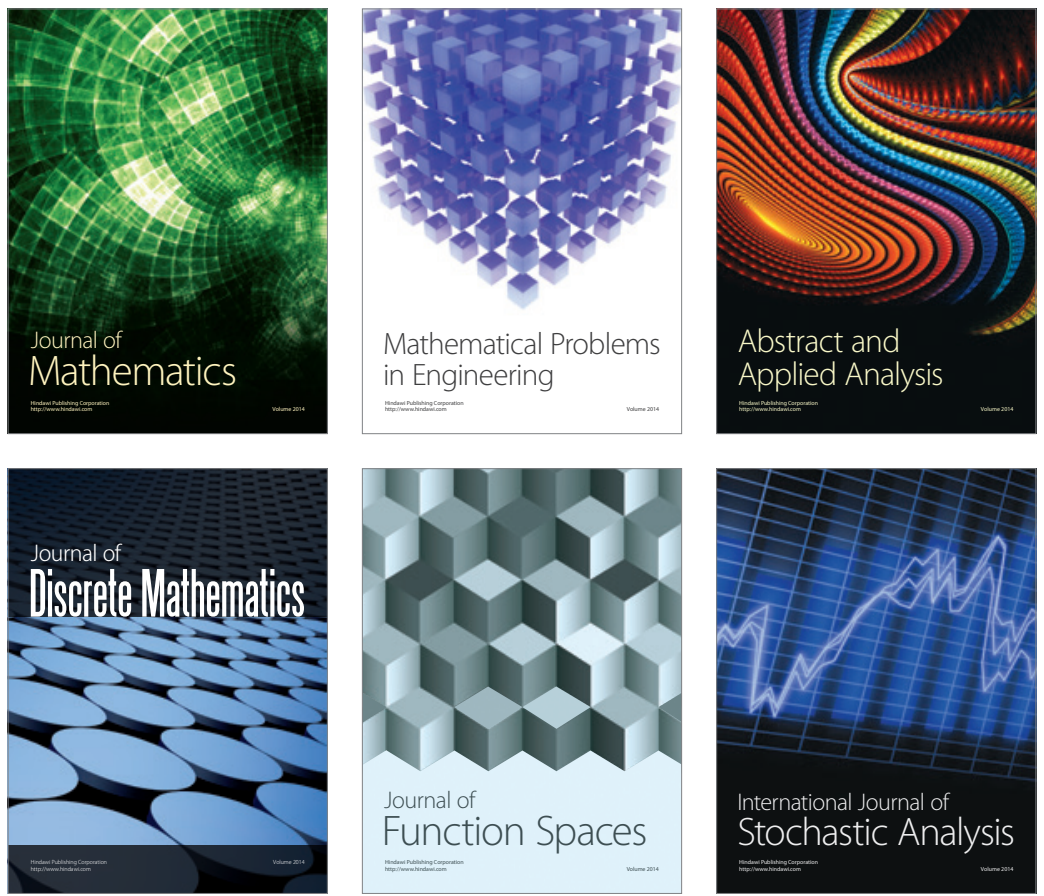

Journal of

Function Spaces

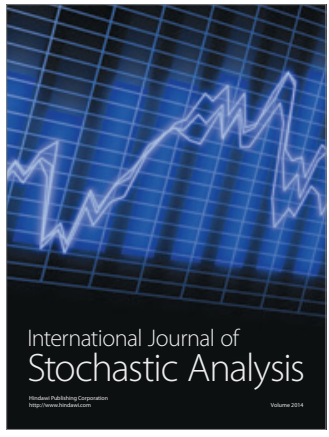

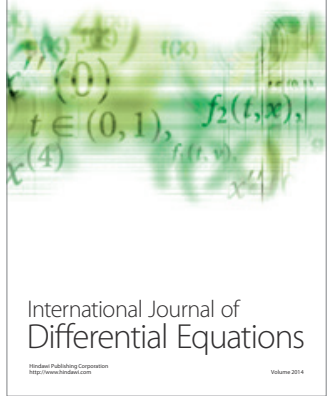
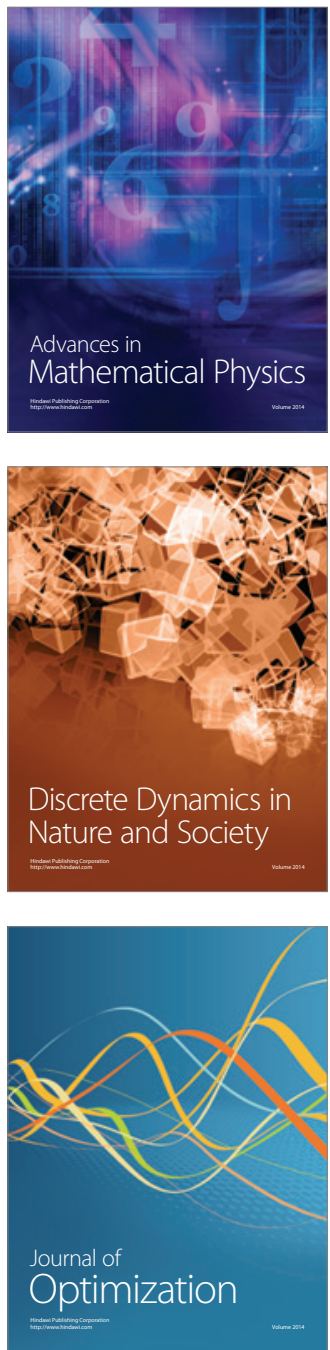